American Journal of Applied Sciences 8 (10): 997-1003, 2011

ISSN 1546-9239

(C) 2011 Science Publications

\title{
A Framework of Successful Executive Information System Development for Education Domain
}

\author{
Mahamsiatus Kamaruddin and Rozilawati Razali \\ Centre of Software Technology and Management, \\ Faculty of Information Science and Technology, \\ University Kebangsaan Malaysia, \\ 43600 UKM Bangi, Selangor, Malaysia
}

\begin{abstract}
Problem statement: Education management is a centralized process of planning, monitoring and controlling entities involved in education affairs so that they align with educational aspirations. It normally concerns several layers of administration that requires different types of information from various operational units. Operational units in education however are scattered and managed independently. As a result, most information is isolated where it is not easily and readily available. This hinders executives from making good judgment since a holistic view about the operations cannot be obtained instantly. Approach: This issue can be resolved, for instance, by having an Executive Information System (EIS). EIS is a dedicated information system that has the capacity to not only retrieving and storing information but also performing comparative analyses through its mapping and merging feature. EIS however does not depend entirely on technology. To succeed, an EIS should be developed based on concrete underlying bases that consider both operational and business aspects. The bases are called Critical Success Factors (CSF). This study discusses the CSF that influence EIS development for education domain. The factors were identified through reviews of related work and two iterations of field work that used semi-structured interviews as the means of data collection. The study adopted qualitative approach where the data were analyzed by using content analysis. Results: The collated CSF form a framework of successful EIS development, which can be employed by education stakeholders as a guideline for developing EIS in the institution. Conclusion: The framework is seen as feasible and practical to be applied in education domain as it considers the necessary elements of education business.
\end{abstract}

Key words: Critical Success Factors (CSF), Executive Information System (EIS), education management, EIS development

\section{INTRODUCTION}

Education Management (EM) is a collective process that recognises, retains, stimulates and unites resources in order to meet educational goals. As a management discipline, EM involves planning, organising, leading and gaining cooperation from the parties involved. It normally comprises several hierarchical levels; tactical or managerial and operational. The top level is responsible of managing and coordinating the administration of the lower levels. It needs to monitor activities and performs comparative analysis so that the overall achievement of educational operations can be determined.

The segregation of educational units based on hierarchical levels causes the information to be isolated and disintegrated. Each unit manages its own respective data by using specific bespoke ways. On the other hand, the ability to access a variety of information is an important asset and has a significant effect on the organisational productivity (Diez and McIntosh, 2009). An executive has to access reliable information in order to decide and create new knowledge. The knowledge is necessary to improve and strengthen performance and services of the organisation.

Computer technology is capable of assisting people in managing their businesses. It has the capacity to acquire information from everywhere and maximise its use. To overcome disintegration issue in EM, one possible technology that can be adopted is Executive Information System (EIS). EIS allows executives to plan, execute and monitor processes and products delivered. EIS

Corresponding Author: Rozilawati Razali, Centre of Software Technology and Management, Faculty of Information Science and Technology, University Kebangsaan Malaysia, 43600 UKM Bangi, Selangor, Malaysia 
offers a method to merge and map information from various sources into one holistic view. This feature is particularly useful for executives to plan options and strategies for future actions (Ikart, 2005; Al-Zhrani, 2010).

Having an EIS to manage education matters seems to be a promising idea. Nonetheless, little is known on how to ensure such an effort can be successful. EIS development does not depend on organisational plans and technology alone (Ikart, 2005). Although the system design can be realised by literally complying the standard system development life cycle, one cannot confirm the success of the overall system (Butler and Fitzgerald, 1999). In essence, EIS development should comprise elements that are not only related to its architecture but also associated with the dimensions of the organisation (Hwang et al., 2007). Studies on information systems such as EIS should focus on the contributing elements that can influence the success of its development (Watson and Rainer, 1996). Identifying and understanding those elements have thus become a central concern.

This study aims to address the above issue by discussing the elements that are necessary for ensuring the success of EIS development for EM. The elements, which are called Critical Success Factors (CSF), were identified through literature reviews and a series of field work that used Malaysia as a context. The study later proposes a framework that describes how the identified elements interact and influence each other.

The study is organised as follows: The following section provides brief background information on EIS and CSF in EIS. It is then followed by the methodology as well as the findings. Later, the proposed framework is described. Finally, the last section concludes the study with a summary of the main findings and future work.

Background: Over the years, the integration of computer technology and information is seen as a strategic platform that can support many management tasks (Cassidy, 2005). The internal and external information are important sources to determine and compare the alternative solutions for the organisational planning (Walters et al., 2003). Attention should be given on that information, which should be in a system that can be extracted to assist the executives to make decisions, plan and organise future directions (Cassidy, 2005). Such a system is called Executive Information System (EIS). EIS aims to improve the ability of decision makers to make a decision based on various sources of information. Realising its importance, many institutions invested in EIS projects to strengthen and improve the efficiency of their management.
An EIS is a sophisticated system, which is widely used in almost every domain including banking (Batiha, 2006), engineering (Hwang et al., 2007) and agriculture (Asadi et al., 2008). EIS is an instance of information systems that contains Business Intelligence (BI) elements (Rasmussen et al., 2009). Through BI, EIS offers the use of dashboard or scorecard features that allow executives to analyse performances from several different viewpoints (Liang and Miranda, 2001). It improves leadership credibility by assisting executives to make decisions based on a computerised system, as compared to manual reports. EIS in short is an integrated information system that has specific structure and features to enhance organisational decision making capabilities. EIS can work flexibly with the data analysis management and incorporates with the end-user through an interactive process (Turban et al., 2010).

Every development aims towards specific objectives, which can be achieved if its respective Critical Success Factors (CSF) is satisfied. CSF is a kind of significant factors that must be considered to reach organisational goals (Seify, 2010). CSF in EIS means the identification of components that are critical for a successful development of EIS (Salmeron and Herrero, 2005). Studies on CSF of EIS need to investigate aspects such as human resources, activities and system as well as environmental factors (Kaniclides and Kimbles, 1995; Salmeron and Herrero, 2005).

\section{MATERIALS AND METHODS}

The study was qualitative research. Qualitative research can be defined as an inquiry process that is executed in real settings in order to understand a certain issue based on comprehensive and complex respondents' views (Krippendorff, 2004). The main purpose of this study was to identify the CSF that influence EIS development for EM. The identified factors acted as the basis for the proposed framework. The factors were generated by considering both theoretical and empirical perspectives.

In general, this study aimed to answer the following research questions:

- What are the CSF of EIS development for EM?

- How can the identified CSF be conceptualised as a feasible framework of EIS development for EM?

The study was based on literature reviews and a series of field work, which were conducted by using 
semi-structured interviews. Figure 1 shows the activities involved. The procedure consisted of three main steps as follows:

Step 1: The identification of research problems and objectives through literature reviews. The literature search was conducted online that covered both journals and proceedings from year 1995. Several articles on CSF for general EIS were found, however none discussed CSF of EIS for EM. This indicates that there is a need for such a study. The interview questionnaires were constructed based on the identified research problems and objectives as well as the underlying principles of EIS and CSF obtained from literature. The questionnaires encompassed issues and items related to EIS development, particularly in education domain. The questions covered four major aspects: software applications in the organisation that support decision making, software development process, EIS development and CSF of EIS development. The questions were constructed in general to limit the effect of personal bias.

Step 2: The execution of the field work. Before the interviews were conducted, the validity and accuracy of the questionnaire were confirmed by two experts in the field of EIS. The interviews were done in two iterations within a period of two year. The first iteration involved several officers at the tactical or managerial level. Later, several representatives from the operational levels were selected in the second iteration. The latter was intended to assess and confirm the reliability of the items identified in the former. The interviews comprised individual (one respondent at a time) and focus group (more than one respondent) sessions.

Step 3: The analysis phase where the findings from the reviews and interviews were examined by using content analysis approach. Basically, content analysis is a systematic way to clarify the content of interview transcripts or related materials and documents (Krippendorff, 2004). Content analysis was chosen because it helped to answer the questions of what and how of the study. The answers led to the identification of CSF and the formulation of framework of EIS development for EM.

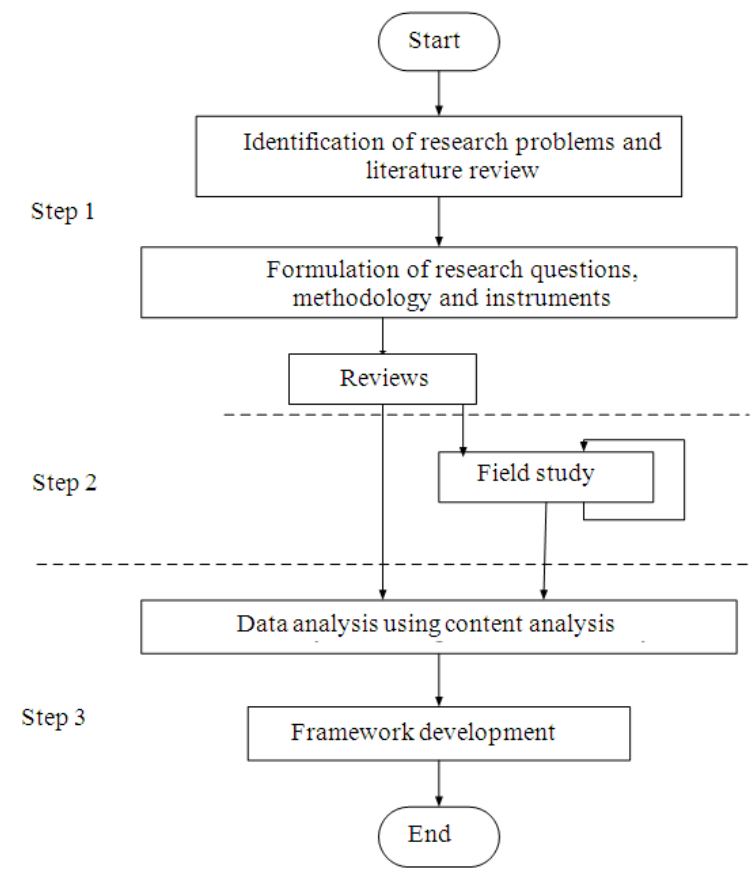

Fig. 1: Research design

Participation: As the domain was EM, the selected respondents were the officers involved in EIS development for education business. The respondents were selected through relevance (purposive) sampling. Relevance sampling aims at selecting specific entities that contribute to answer a given research question (Krippendorff, 2004). There were eleven respondents altogether. The number may be considered as small but it was seen as sufficient since the population was identical. Moreover, the respondents were experts in the domain.

The first iteration of interview was completed at the Ministry of Education (MOE). The respondents comprised four officers from MOE and one senior officer from a government agency. MOE officers are considered as experts and stakeholders in EM. Although the sole respondent from the government agency was not from the domain, the person was an expert in EIS development. The details of the first iteration can be found in earlier study.

The second iteration of interview was conducted at two public universities and MOE respectively. The participation from universities was included since they are also dealing with education matters. Moreover, universities also need integrated management systems to administer their businesses (Oliveira et al., 2011). Table 1 depicts the background of the respondents who took part in the study. 
Am. J. Applied Sci., 8 (10): 997-1003, 2011

Table 1: Respondents' background

\begin{tabular}{llll}
\hline Iteration & Agency & Respondent & Designation \\
\hline Phase 1 & MOE & Respondent 1 and 2 & Head of unit \\
& MOE & Respondent 3 and 4 & Officer \\
& Agency A & Respondent 5 & Officer \\
Phase 2 & MOE & Respondent 6 and 7 & Officer \\
& University A & Respondent 8 and 9 & Officer \\
& University B & Respondent 10 and 11 & Officer \\
\hline
\end{tabular}

Note: Agency A-Government Agency; MOE-Ministry of Education

Table 2: CSF of EIS development for education management

\begin{tabular}{|c|c|c|c|}
\hline Factor & Theme & Reviews & $\begin{array}{l}\text { Field } \\
\text { study/work }\end{array}$ \\
\hline \multirow[t]{7}{*}{ People } & Executives/top management & & \\
\hline & Support and involvement & I & I \\
\hline & Development team & & \\
\hline & Technical skills & I & I \\
\hline & Interpersonal skills & $\mathrm{x}$ & I \\
\hline & Education personnel & & \\
\hline & Information management & I & I \\
\hline \multirow[t]{11}{*}{ Process } & Development activities & & \\
\hline & Development methodology & I & I \\
\hline & System requirements & I & I \\
\hline & System maintenance & I & I \\
\hline & Monitoring (follow-up) & $\mathrm{x}$ & I \\
\hline & Data extraction & & \\
\hline & Periodically & I & I \\
\hline & Adequate & $\mathrm{x}$ & I \\
\hline & Correct & l & I \\
\hline & Data management & I & I \\
\hline & $\begin{array}{l}\text { Hardware and software } \\
\text { infrastructure }\end{array}$ & l & I \\
\hline Product & $\begin{array}{l}\text { Integrated features } \\
\text { and functions }\end{array}$ & l & I \\
\hline \multirow[t]{15}{*}{ Environment } & Plan/Policy & & \\
\hline & Education strategic plan & $\mathrm{x}$ & I \\
\hline & Key Performance & $\mathrm{x}$ & I \\
\hline & Indicators (KPI) & & \\
\hline & Standard Operating & $\mathrm{x}$ & I \\
\hline & Procedure (SOP) & & \\
\hline & Project management & I & I \\
\hline & Management best practices & & \\
\hline & Financial & I & I \\
\hline & System awareness & I & l \\
\hline & IS sources & I & I \\
\hline & Politics and culture & & \\
\hline & $\begin{array}{l}\text { Cooperation to } \\
\text { information sharing }\end{array}$ & l & l \\
\hline & Attitude towards projects & I & I \\
\hline & $\begin{array}{l}\text { Organisation culture } \\
\text { towards projects }\end{array}$ & l & I \\
\hline
\end{tabular}

Note: Review-5 articles; Field study/work-2 iterations

\section{RESULTS}

The content analysis approach was used to identify CSF of EIS development for EM. The content analysis is a directed approach. With a directed approach, the analysis starts with a theory or relevant research findings as guidance for initial codes (Hsieh and Shannon, 2005). Later, the codes are categorised using both manifest and latent content. The former is the specific, clear, surface contents that are easily categorised whereas the latter refers to the underlying meaning contained in a reference text.

The results were analysed by using a qualitative analysis tool: Nvivo. Nvivo is suitable for running qualitative content analysis of document literature and interview transcripts. In order to identify the factors (manifest) and their respective themes and items (latent), deductive and inductive techniques were adopted in the analysis procedure.

By applying the deductive technique, four CSF were identified: organisational settings (Environment), human resources (People), development activities (Process) and EIS itself (Product). These factors are similar with the ones discovered by previous studies (Kaniclides and Kimbles, 1995; Salmeron and Herrero, 2005). The factors were divided into themes and items through the inductive technique. For example, the Environment factor was divided into three themes in order to illustrate succinctly the environmental factors that affect EIS development. The three themes were further elaborated by the respective items.

Two main sources were used as the basis for the analysis: reviews of related articles and field work. The reviews were based on articles concerning CSF of EIS development that were published within a period of fifteen years. The keywords used in the searching were "critical success factors" and/or "executive information system" as well as "education management". There were quite a number of related articles found. However, only five studies covered both CSF and EIS (Al-Shidadi, 1997; Bajwa et al., 1998; Srivihok, 1999; Poon and Wagner, 2001; Salmeron and Herrero, 2005). These studys in fact discussed CSF for general EIS development, particularly for profit-based organisations. None of the articles investigated CSF of EIS development for education management purposes. Those five articles were thus chosen as the objects for comparing with the field work because they suited best with the interest of the study. On the other hand, the field work involved eleven respondents from two iterations of investigation, as described in earlier section. Table 2 below shows the findings from both sources, which contains CSF of EIS development for EM. The CSF and their respective themes were found in both reviews and field work. Most items for the themes were present (marked as/) in both sources except six 
items (marked as $\mathrm{x}$ ) emerged only from the field work. As the study concerned the domain, priority was given towards the field work. An item was considered for further analysis if it appeared in the interview transcripts more than once, although it was not found in the literature reviews.

In order to confirm the findings, a reliability test was performed on the items gathered from the two iterations of field work. The reliability test is useful for qualitative study because researchers can be more certain of counts any particular theme is mentioned in the reference text (Bernard and Ryan, 2009). The agreement is based on Cohen's kappa or k value. The acceptable level of agreement is when the $\mathrm{k}$ value is between 0.61 and 0.80 (Bernard and Ryan, 2009). The $\mathrm{k}$ value for this study is 0.75 . This indicates that the items are adequate and reliable and thus can be included in the proposed framework.

\section{DISCUSSION}

The CSF and the respective themes and items discovered in earlier analyses were conceptualised as interrelated entities to form a framework of EIS development for EM, as illustrated in Fig. 2.

The success of EIS development for EM is influenced by the managerial and technical people who need to collaborate during the development process in order to produce an integrated EIS product. These factors ought to be supported by the organisation's environment.

Environment factor involves the management practices that coordinate EIS development until the system can fully be used. Management needs to acquire and plan allocation for the EIS project, conduct awareness programmes and identify information sources, which should be integrated in the EIS product. These activities cannot be accomplished by the management solely. Cooperation among education personnel in terms of information sharing culture and positive attitude towards the project and system should be obtained. The parties involved in the project have to consider educational strategic plans, Key Performance Indicators (KPI) and Standard Operating Procedures (SOP) as well as project management policies during the process.

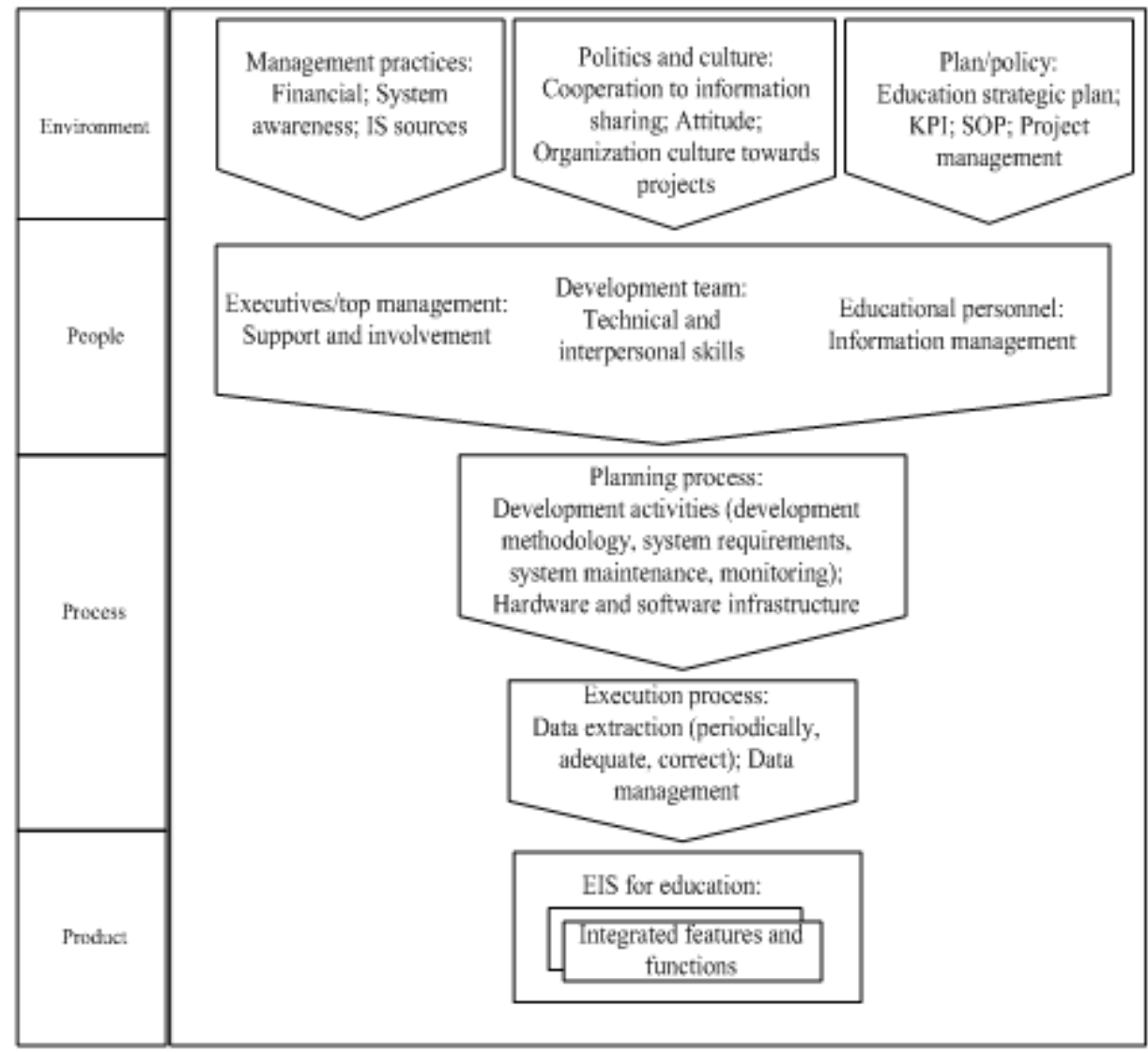

Fig. 2:Framework of EIS development for education management 
People factor includes the organisation's top management or executives, EIS development team and education personnel. These parties need to understand the purpose and importance of an EIS project in improving organisational planning and decision making. An EIS project cannot succeed without strong support and involvement from the executives, besides financial and other tangible resources. The support is particularly necessary for handling political and user resistance towards the system. The EIS development team on the other hand, should possess technical and interpersonal skills when building the system. The technical skills are needed for the operational part, whereas interpersonal skills are required for getting right requirements and obtaining users' acceptance. The development team is responsible to plan, develop and maintain the technical infrastructure and manage the system operations. The education personnel act as the sources of information where their primary contribution is to provide adequate and correct data into the system timely. They need to manage the information properly so that it becomes reliable. Reliable information leads to meaningful analysis and thus enhances decision making by the executives.

Process factor includes the planning and execution activities that happen throughout EIS development. The planning process must take into account the development lifecycle as well as hardware and software infrastructure. The execution process has to synchronise data extraction and data management activities for various information sources.

Product is the developed EIS that contains integrated features and functions concerning education business. The system should continue to change or evolve over time in response to new user and business needs.

\section{CONCLUSION}

Decision making is a critical action. Any decision made has an impact to an organisation either in short or long term. The use of technology can improve the management, dissemination and reporting of information for decision making purposes. The technology however only provides the infrastructure. It cannot guarantee the success of a system development. On the other hand, each domain has a unique process. Therefore, there is a need to identify the contributing factors that affect a system development for a particular business domain.

This paper has discussed the Critical Success Factors (CSF) of Executive Information System (EIS) development for educational domain. The CSF has been identified through literature reviews and two iterations of field study involving domain experts as well as personnel who were involved in EIS projects. There are four CSF altogether, namely People, Process, Product and Environment. Each of the CSF comprises a number of elements that have different impacts on the success of EIS development. In the study, the CSF has been integrated as interrelated elements to form a framework of EIS development for managing education affairs. The framework may not be exhaustive where it can be validated and refined further in future by replicating the study through the use of prototype as a proof of concept. However, it provides some directions towards a successful EIS development for education domain.

\section{ACKNOWLEDGEMENT}

The researchers thank the respondents participated in the study. This study was funded by the Ministry of Education, Malaysia.

\section{REFERENCES}

Al-Shidadi, A.J., 1997. Factors influencing the success of the EIS implementation. Admin. Sci. J. J. King Saudi Univ., 9: 39-59.

Al-Zhrani, S., 2010. Management information systems role in decision-making during crises: Case study. J. Comput. Sci., 6: 1247-1251. DOI: 10.3844/jcssp.2010.1247.1251

Asadi, A., A. Rezaei and A. Rezvanfar, 2008. Improvement Mechanisms of Management Information System (MIS) In Iran's agricultural extension organization. Am. J. Agric. Biol. Sci., 3: 462-467. DOI: 10.3844/ajabssp.2008.462.467

Bajwa, D.S., A. Rai and I. Brennan, 1998. Key antecedents of executive information system success. A Path Analytic approach. Decision Support Syst., 22: 31-43. DOI: 10.1016/S01679236(97)00032-8

Batiha, K.M., 2006. An EBA-web based application for banking information system. J. Comput. Sci., 2: 889-895. DOI: $10.3844 /$ jcssp.2006.889.895

Bernard, H.R. and G.W. Ryan, 2009. Analyzing Qualitative Data: Systematic Approaches. 1st Edn., Sage Publication Inc., USA., ISBN-13: 9780761924906, pp: 480.

Butler, T. and B. Fitzgerald, 1999. Unpacking the systems development process: An empirical application of the CSF concept in a research context. J. Strategic Inform. Syst., 8: 351-371. DOI: 10.1016/S0963-687(00)00027-5

Cassidy, A., 2005. A Practical Guide to Information Systems Strategic Planning. 2nd Edn., Auerbach Publications, ISBN-13: 978-0849350733, pp: 400. 
Diez, E. and B.S. McIntosh, 2009. A review of the factors which influence the use and usefulness of information systems. Environ. Model. Software, 24: 588-602. DOI: 10.1016/j.envsoft.2008.10.009

Hsieh, H.S. and S.E. Shannon, 2005. Three approaches to qualitative content analysis. Qualitative Health Res., 15: 1277-1288. DOI: 10.1177/1049732305276687 PMID: 16204405

Hwang, W.T., S.W. Tien and C.M. Shu, 2007. Building an executive information system for maintenance efficiency in petrochemical plants. Trans. I Chem. E Part B, Process Safety and Environ. Protection, 85: 139-146. DOI: 10.1205/psep06019

Ikart, E.M., 2005. A Theory-based Model for the Study of Executive Information Systems Adoption by the Top-level Managers. http://www.pacisnet.org/file/2005/295.pdf

Kaniclides, A. and C. Kimble, 1995. A Framework for the Development and Use of Executive Information System. Proceedings of GRONICS, Groningen, The Netherlands, pp: 47-52.

Krippendorff, K.H., 2004. Content Analysis: An Introduction to its Methodology. 2nd Edn., Sage Publications, USA., ISBN-13: 978-0761915454, pp: 440.

Liang, L.Y. and R. Miranda, 2001. Dashboards and Scorecards: Executive Information Systems for the Public Sector.

Oliveira, L., N. Vasconcelos, F. Queiroz, J. Queiroz and H. Hékis, 2011. Contribution of integrated management systems to university management: case study of the federal university of Rio Grande Do Norte. J. Soc. Sci., 7: 415-422. DOI: 10.3844/jssp.2011.415.422

Poon, P. and C. Wagner, 2001. Critical success factors revisited: success and failure cases of information systems for seniors executives. Decision Support Syst., 30: 393-418. DOI: 10.1016/S01679236(00)00069-5
Rasmussen, N.H., C.Y. Chen and M. Bansal, 2009. Business Dashboards: A Visual Catalog for Design and Deployment. 1st Edn., John Wiley and Sons, Inc., ISBN-13: 978-0470413470, pp: 304.

Salmeron, J.S. and I. Herrero, 2005. An AHP-based methodology to rank critical success factors of executive information systems. Comp. Standard Interfaces, 28 : $1-12$. DOI: 10.1016/j.csi.2004.09.002

Seify, M., 2010. Importance of KPI in BI system, case study: Iranian Industries. Proceedings of the 7th International Conference on Information Technology, Apr. 12-14, Las Vegas, Nevada, USA., pp: 1245-1246. DOI: 10.1109/ITNG.2010.238

Srivihok, A., 1999. Understanding executive information systems implementation: An empirical study of EIS success factors. Proceedings of 32nd Hawaii International Conference on System Sciences, Jan. 05-08, Maui, Hawaii, pp: 1-10. DOI: 10.1109/HICSS.1999.772794

Turban, E., R. Sharda and D. Delen, 2010. Decision Support And Business Intelligence Systems. 9th Edn., Pearson Prentice Hall International, Inc., ISBN: 978-0136107293, pp: 696.

Walters, B.A., J. Jiang and G. Klein, 2003. Strategic information and strategic decision making: The EIS/CEO interface in smaller manufacturing companies. Inform. Manage., 40: 487-495. DOI: 10.1016/S0378-7206(02)00063-0

Watson, H.J. and R.K. Rainer, 1996. Building Executive Information Systems and Other Decision Support Applications. John Wiley and Sons, Canada. ISBN: 978-0471069300, pp: 512. 